\title{
Nutrition Rehabilitation Centers and Locally Prepared Therapeutic Food in the Management of Severe Acute Malnutrition PEDIATRICIAN'S PERSPECTIVE
}

\author{
KE ELIZABETH \\ Consultant in Nutrition and Genetics, Professor of Pediatrics and Superintendent, \\ SAT Hospital, Govt. Medical College, Thiruvananthapuram, Kerala-695011, India. \\ elizake@hotmail.com
}

T Wwenty years after initiating 'Breastfeeding Policy' in 1992 and 10 years after initiating the Global Strategy for 'Infant and Young child feeding' (IYCF) in 2002, we have to admit that millions of under-five children in India suffer from Moderate acute malnutrition (MAM) and Severe acute malnutrition (SAM) including edematous SAM(E-SAM). As per the NFHS-3 (2005-06) data, 43\% are underweight, $48 \%$ are stunted, $20 \%$ are wasted and $7.9 \%$ have severe wasting [1]. Due to the association of malnutrition with morbidity and mortality, specialized treatment and preventive interventions are required. SAM cases with medical complications need to be managed and followed up in nutrition rehabilitation centers (NRCs). Active case finding of the underweight children, utilizing growth monitoring chart available in the Mother Child Protection (MCP) Card and categorization as per weight for age (underweight), height for age (stunted) and weight for height (wasted) are required for appropriate intervention.

Malnutrition is usually noted in the stereotypic profile of poverty, ignorance and illiteracy or natural calamities like war or famine but currently causal factors like 'manmade perception' of lactation failure, abrupt breastmilk withdrawal and early introduction of diluted formulae/ diets are being increasingly identified [2]. Malnutrition being a 'Bio-Psycho-Social Disorder', biologic/organic causes, psychological disturbances in individuals/family and or social factors are causative, and not necessarily lack of food alone.

NRC is a unit in a health facility where children with SAM are admitted as per the defined admission criteria and provided with medical and nutritional therapeutic care $[3,4]$. Discharge from NRC is to be based on attainment of target weight-gain of $15 \%$ from the admission weight and the child should continue to be in the nutrition rehabilitation program till she/he attains the cure weight of $>1$ SD or $90 \%$ of the expected weight-for-height. In
NRCs, in addition to curative care, special focus should be on timely feeding, adequate and appropriate for children and on improving the skills of mothers / caregivers on optimum age appropriate caring and feeding practices. Efforts are also to be made on capacity building through counseling and practical demonstrations on infant feeding, sensory stimulation, play therapy, imparting tender loving care and so on. The ambience of the NRC should be warm, colorful and attractive.

In-patient management of children with SAM is highly effective in reducing case fatality rates, but even under the best circumstances inpatient facility will not be able to handle the entire caseload in a given high priority area. Most states of India already have many NRCs, but some had none; Kerala recently started the first NRC in the tribal belt of Attappadi, Palakkad District in June, 2013. Other issues with respect to NRCs are long hospital stay requiring the mother/caregiver to stay away from home and the father missing work for many days, and the resultant practice of leaving against medical advice.

Implementation of the 10 steps of SAM management protocol often need to be modified as per the availability of proxy preparations of F75, F100 and RUTF that are adapted by NRCs in India [5]. Imported feeds of the above preparations are considered not acceptable, feasible, affordable and sustainable in the Indian setting. Several proxy feeds based on whole strength milk, infant formula, skimmed milk powder, cereal flour etc. are being tried in various NRCs in India [6-8].

Outcome measures of NRCs include survival, default, discharge and cure rates $(3,4)$. High default rates and low cure rates are of great concern in the present setting. 12 NRCs from UP have reported very low mortality rate $(1.2 \%)$ and an average weight gain of $12.1 \mathrm{~g} / \mathrm{Kg} / \mathrm{day}$, which is commendable, but it is also reported that $53 \%$ had to be 
discharged before recovery/attaining target weight for discharge, and default rate was as high as $47 \%$ (5). Similar observation has been made from other facilities as well [8]. Recent experience from a tertiary care teaching hospital was: $40.3 \%$ cure, $13.4 \%$ default and $5.9 \%$ mortality with 3 deaths out of the 67 enrolled occurring before getting initiated on SAM management [7].

A community-based program for the management of SAM without medical complications and for those who are to be followed up after NRC care should be in place to complement the services of NRCs. Moreover, each child should be line listed and tracked in the community before and after discharge using the available health care/ICDS/ ASHA workers and the system of Mother Child Tracking System (MCTS)/mobile phone SMS. Research and development, and consensus on locally prepared therapeutic food proxies as used in regional NRCs should be considered as a national priority.

Funding: None; Competing interests: None stated.

\section{REFERENCES}

1. International Institute for Population Studies (IIPS) and Macro International. National Family Health Survey (NFHS -3), 2005-2006. IIPS, Mumbai, India, 2007.
2. Elizabeth KE. Changing profile of undernutrition and edematous severe acute malnutrition (E-SAM). Indian Pediatr. 2012;49:843-4.

3. World Health Organization (WHO) Guidelines for Inpatient Treatment of Severly Malnourished Children. WHO, Geneva, Switzerland, 2003.

4. Indian Academy of Pediatrics (IAP). IAP Guidelines 2006 for hospital-based management of severly malnourished children (adapted from WHO Guidelines). Indian Pedaitr. 2007;44:443-61

5. Singh K, Badgaiyan N, Ranjan A, Dixit HO, Kaushilk A, Kushwaha KP, et al. Management of children with severe acute malnutrition: Experience of nutrition rehabilitation centres in Uttar Pradesh, India. Indian Pediatr. 2014; 51:21-5.

6. Kapil U. Ready to Use Therapeutic Food (RUTF) in the management of severe acute malnutrition in India. Indian Pediatr. 2009;46:381-2.

7. Elizabeth KE. Adoption of WHO Protocol for Hospital Based Management of Severe Acute Malnutrition (SAM) in Underfive Children - An Observational Study. SAT Endowment Scheme Project Report. SAT Hospital, Govt. Medical College, Thiruvananthapuram, 2013.

8. Namidi RS, Kulkarni B, Radhakrishna KV, Shatrugna V. Hospital based nutrition rehabilitation of severely malnourished children using energy dense local foods. Indian Pediatr. 2010;47:687-93. 